\title{
Ergonomic analysis for elementary school teachers. Preliminary trial for elementary schools in the province of Buenos Aires
}

\author{
Gabriela Cuenca ${ }^{\mathrm{a}, *}$, and Patricio Nusshold ${ }^{\mathrm{b}}$ \\ ${ }^{a}$ Departamento de Ingeniería Industrial, Facultad Regional Buenos Aires, Universidad Tecnológica Nacional, \\ Argentina.info@gabrielacuenca.com.ar \\ ${ }_{b}$ Secretaría de Investigaciones, Facultad de Psicología, Universidad de Buenos Aires, Argentina. patri- \\ cio.nusshold@gmail.com
}

\begin{abstract}
This article presents the results of a preliminary trial, in the context of an intervention, developed by a team of ergonomists under the request of the Occupational Health Department of the General Management of Schools in the Province of Buenos Aires, Argentina. We present a reformulation of the request and the results obtained in 2009 from observations and interviews held in three schools in the province of Buenos Aires in which different jobs positions were assessed. From this stage onwards, the tools were validated and new aspects were shown, particularly in relation to the high variety of real situations in the education sector.
\end{abstract}

Keywords: teachers, education, ergonomics, work analysis, methodology

\section{Introduction}

The following research has been done under the request of the Occupational Health Department of the Culture and Education Ministry of the Province of Buenos Aires. The initial request was to design different job profiles and through these get to know: the current work conditions of primary school workers of Buenos Aires, employment-related pathologies and their effects on leaves of absence. A series of meetings were held in order to reformulate the demand to include a view of the work conditions from the ergonomics point of view.
By studying the work situations of the schools, we sought to understand the work context, the interactions between the worker and the tools he used to undertake his position, the dynamics of the worker's activity, the variations in the activity and in the conditions of the worker, as well as the variations in his working environment.

The aim for this first stage of the preliminary trial was to validate the instruments of the investigation, and to highlight, through observations and interviews, the complexity of the real situation.

- $\quad{ }^{a}$ Ergonomist, Industrial Designer,Departamento de Ingeniería Industrial, Facultad Regional Buenos Aires, Universidad Tecnológica Nacional, Argentina.info@gabrielacuenca.com.ar

- $\quad{ }^{b}$ PhD Student, Ergonomist, Organizational Psychologist, Secretaría de Investigaciones, Facultad de Psicología, Universidad de Buenos Aires, Argentina.patricio.nusshold@gmail.com

- $\quad$ Both authors are members of the Asociación de Ergonomía Argentina. Departamento de Salud Ocupacional, Dirección de Cultura y Educación de la Provincia de Buenos Aires y de la Facultad de Medicina, de la Universidad Nacional de La Plata 
Initially, the team conducted a literature review of the studies of ergonomics, occupational health and safety, and work psychology and psychodinamics of the work conditions in the education sector.

In each school during the preliminary trial (first stage of the project) interviews were held with the director, the secretary, three teachers of different levels, a teacher assistant, and the librarian. Personal interviews were conducted and a registry of inventories was undertaken. On site observations were made and the real work situation was filmed, both during class as well as during class breaks.

The first stage of the research project focused on the design, assembly, and adequacy of the existing analysis methods; methods which are well known and considered standards in the scientific world of ergonomics.

The selected methods were:

For the analysis of psychosocial and mental factors: ISTAS 21, the Job Satisfaction Scale (Warr, Cook \& Wall, 1973) and the Psychosocial Factors Questionnaire, identification of risk situations, of the Institute of Occupational Safety Navarro, 2002.

To analyze occupational discomfort: the Discomfort Nordic Questionnaire was adapted to this research investigation. To analyze the working conditions and environment the CyMAT Survey 1986 of the ILO was used.

\subsection{Activity Ergonomics and the state of art on School Workers' Health.}

The following research is focused on the analysis of teacher's position in the public primary schools of the province of Buenos Aires, in Argentina. Director and auxiliary positions have also been assessed.

Political, economic and social changes, as well as a low budget assigned to education and infrastructure, have historically determined a process of impoverishment of work conditions, an increase of the quantity of working hours, pathologies and occupational diseases and a low social recognition on the role of teachers. When this recognition does not exist (due to ignorance which minimizes the importance of the task) it becomes very difficult to improve work conditions, as society hold an understanding that educational workers do not work very hard.

It is not evident how to improve work conditions and change the established preconception in society. Also, the tasks of the educator have been altered and other tasks are now included for which workers are
Guided tours of the schools were also conducted with the intention of trying to understand the roles and objectives of each professional interviewed. However, it must be noted that participation of the survey was not compulsory.

Due to the size of the sample, the conclusions made in this paper can only seek to refine the tools and identify certain features, and conclusions cannot be extrapolated to the entire primary school working population. However, some clues to the continuity of the project became evident.

Once the preliminary trial was completed we were able to observe that the designed tools were valid to assess not only the physical settings but also the workload and associated job stress. However, only through interviews and onsite observations were we able to analyse how real life situations affect each job position. The situations are presented with a high level of variability not only from one job position, or one school, to another but also within the same school group. The gap between tasks, objectives and functions (defined by extremely rigid guidelines) for each of the jobs, and what was observed in reality was quite significant. Therefore, to ensure successful educating, it appears that workers constantly have to adapt themselves to changing conditions and confront different and often unforeseen challenges in the workplace.

not prepared physically, psychologically or professionally. Amongst these new tasks we could find serving lunch, understanding social cases or dealing with aggressions of parents and children.

The medical and psychiatric absences are administered independently of the real work situation. Sometimes, abuses of certain type of absence permissions take place, or on the contrary, sometimes workers still not ready are being forced to return in advance. The majority of the occupational diseases are caused by the organization of work, an aspect that is not sufficiently considered when treating diseases or when re-inserting the worker into the workplace. Therefore, workers are frequently obliged to return to the work place which caused the disorder, in the exact previous harmful conditions.

The treatment of those who take medical absence permissions, as well as the control of the treatments mentioned above, is done without considering the conditions in which the worker got ill or even where they will have to return, once absence finishes.

In addition, the recent middle class population transition from public education towards private edu- 
cation means that a great number of situations that some time ago were solved by parent associations must now be solved by the school workers themselves. The amount of social risk cases that public primary schools have to deal with among student's population have now increased and most of new situations were recently unthinkable.

\subsection{Activity Ergonomics}

The intervention was performed using Activity Ergonomics model, analyzing determinants and consequences of labor activity in order to contemplate how these aspects interact. This would led to ideas for design of work positions in schools that could adjust to workers possibilities and improve both their health and potential increasing results in terms of educational quality.

\subsection{The initial request}

The initial request was to design different job profiles and through these get to know the current work conditions of primary school workers of Buenos Aires, their employment-related pathologies and their effects on absences.

This first request was determined with the team of the medical board of the Occupational Health Department of the General Management of Schools in the Province of Buenos Aires, Argentina.

Afterwards, the initial request was reformulated, since the job profiles were only feasible if the real content of the work role was known, and it was necessary to carry out an ergonomic analysis of the working places of the educational workers.

\subsection{Choosing the schools for the preliminary trial}

A series of meetings were held in order to consider the demand of viewing work conditions from the ergonomics point of view.

The first stage was defined as the undertaking of a pilot test in three schools from the city of La Plata, and it had the aim of testing the protocols of evaluation, and defining the development of the final sample in the province that would include eight cities. The Inspector of the Zone made the choice of the schools. It was decided that the sample would analyze three different types of schools: a rural school, a central city school and a peripheral school. Likewise, it was decided the interviews would be done to: the school director, two teachers and a teacher's assistant or auxiliary. The same scheme would be reproduced after the preliminary trial in the Province of Buenos Aires, where each of the members of the research team would work with the three different types of schools. Adding to the three schools of the preliminary trial, this will give a sample total of 24 schools and about 100 interviews.

This first definition of the preliminary trial is thus presented as central to the development of the general intervention to be undertaken at a later stage. This first stage will not only seek to define the quantitative protocols, but principally be able to place the workers thoughts centrally to our research. Not only an analysis of the work prescription, the tasks, or an observation limited to the physical aspects of the work environment will be done in order to design a job profile, but our intervention will also centre on the way the different workers face the variability of situations they have to deal with. How they deploy their abilities to adapt their work role to the reality. It is, in this process of confrontation between the task and the real situation, between the institutionalized and the possible, that each of the workers of the General Management of Schools are able to ensure the result of education, often falling ill along the way.

\subsection{What we found}

In each of these three schools, multiple examples of the way in which workers (all women in the preliminary trial) were managing at school, and beyond, to apply their aptitudes in order to ensure the employer's expected result (the inspector, pupils and parents, amongst others).

Many singular aspects appeared when analyzing particular situations and most of the verbalizations and observations of the positions turned out to be very interesting. Many aspects that were not relieved by the questionnaires appeared throughout the interviews showing key aspects of these work situations, such as the loneliness suffered by rural teachers, work continuity in spite of being sick in order to keep assumed commitment, directors difficulties to solve infrastructure matters, or the variability of parent associations from one school to another. Social aspects also appear to have a great impact on the activity of school workers: the raise of violence in pupils and parents, the lack of critical tools needed for meeting educational ends or the lack of adequate physical space.

Some aspects that were found equally in three type of schools will be now presented, followed by 
some particular situations that were verbalized in each of three schools: the rural one, the central one and the peripheral one.

\subsection{The common aspects to the three kinds of} schools

Most of the teachers interviewed had two or up to three different positions in the same school or in different ones on a single labor day, being an unfavorable situation since it prevents the possibility of recovery and rest. As for the recess time during work, even though there are established pauses that are those of the playtime of children or when there is a special class, it was found that most of the teachers did not have much time to rest during their working hours, since during the playtime they must be in the courtyard taking care of children or doing another labor and when there is a special class (Music, Art, English Courses) their strategy is to advance in the correction of the pupils' notebooks.

They say that there is not a scheduled time for exchanging experiences with other teachers, advise each other or take a break from different difficulties that may come up within their daily work.

The work to be done is prearranged, and therefore the order of these steps cannot be modified according to the worker's will, since there is a schedule for the activities and a logical sequence to follow. However, there can be small alternatives introduced by the worker.

As for the directors, they also have counter shift tasks and passage of information, which extends their labor day. This is important since they are reviewed for the 4 hours that they are supposed to work, being this inaccurate. Also the remunerations are calculated based on this parameter, and they are certainly missed.

The auxiliaries have two big moments of great intensity and volume of work: at the beginning and in the end of their labor day, having less intensity during class hours.

Until the current labor situation is not thoroughly known and recognized, the dissatisfaction will continue.

The workers performance assessment methodology, especially the director's and teachers', show a great level of variability between one school and another. While the regulation statement can be essential in certain schools, it seems to be secondary to the day to day activities in others. Consequently, the re- spect of schedule will be central in certain schools, whereas in others establishments it would only mean an additional indicator.

Coming up, the three schools that formed the pilot test as an example of the great variability of situations that have been observed in the schools.

Not only the physical aspects of the jobs will be described, but also distinctive aspects of the work organization and qualitative indicators as some verbalizations realized by the workers during the analysis of their activity.

\subsection{The rural school}

The rural school is characterized for being isolated, far in the countryside, with very difficult access when it rains, as to reach it the only way is trespassing 5 kilometers of muddy land.

There are no signs indicating the location of the school, making it hard to be found.

The teacher holds all the functions and roles since she is the teacher of all the pupils, and is also the director and assistant. She also fulfills the functions of coordination and undertaking of the direction of the school, and undertakes the education and the maintenance of cleanliness of the establishment.

With regards to the building, it is a relatively new construction that consists of two parts. In the main one there is a hall of entry, towards the right a double classroom is found with large windows and towards the left side there is the kitchen fully equipped with an electrical refrigerator and gas stove. Behind the kitchen we find two bathrooms for boys and girls and in between a sink used for cleaning and hygiene.

In the other part of the building there is a hall that in the future is planned to be used as a kindergarten. At the present, this space is used as a meeting room.

The school has a population of only 5 pupils. These pupils live in the area, come to the school by their own means, are rarely absent, and they all are in different grades.

The teacher says that the parents are floriculturists and others work in a brick factory near the school. These parents are working during the school hours and therefore do not get to participate in acts of the school or get to follow closely the development of their children's education. It is very important for the parents that their children study so that they can get a better job in the future than themselves.

The classroom is equipped with two blackboards, bookshelves, simple desks and chairs, a ventilator, 
electrical heating and a gas heater, natural lighting and artificial lighting consisting of unprotected fluorescent pipes (the above mentioned are very high and do not give good light). The floor is made of granite, the ceiling is of wood, and there are big large windows with curtains in one of the wings. They also possess a first aid kit and a computer.

The pupils are in different grades, which implies for the teacher generating a particular strategy of education. Every day, the teacher presents a single topic for all the pupils and then she gives an exercise of a different level of complexity according to their age and level to each of the students. This forces her to prepare a different task for each of the 5 pupils.

When pupils are absent, parents come to school to look for the homework or ask other students to take it for them, so their children can work at home.

For each of these children a record must be taken. Every class must be registered, even if there is only a single student in the level. This means that even if she has only five students she must do the paperwork for five levels.

In the rural school, besides the administrative thing and the classroom work, the teacher has other tasks before going to the school:

- She shops for the food for the preparation of the school breakfast.

- She carries the keys of the school (the children wait for her outside). She says " this is why I'm never absent."

Once at school, she : stoves.

Opens the school, in winter she lights the

- Hoists the flag,

- Begins to give lessons.

- $\quad$ Once she gives a task to the pupils, she begins to prepare breakfast what takes her half an hour.

- During the longer break when the pupils go and play outside, she cleans and after the weekends she does a deep cleaning: "I clean like at home"

She maintains good functioning of the electric water pumps. tanks for her.

Receives to the gas truck that loads the gas

- When the water pump does not work she transports the water from town and other times the water cans are brought to her but without the dispenser, which means that she must load manually the water can and distribute it for everything what can be used (cleanliness, domestic use) etc.

Some interesting comments of the rural school teacher were:

"The culture of the school is a "family" culture, the pupils never miss class, arrive by themselves on bicycle, the school is like a home, the parents don't come to the school for events and do not take part actively, but when one of the children is sick they do not want them to miss the material of the classes, do not want their children to be like they are, many parents say "I want my son to progress, and not to be employed in a brick factory or a country house like me."

"The deficiencies that I possible have at school are made up by the quality of the children and the people of the neighbourhood; I am in love with this school"

Nevertheless, she does not forget all the overdue work that she has, as she says "the bureaucracy overwhelms me, senseless papers but I still have to fill them in, it is difficult for me to engage in further training as I do not have time. "

In addition, it turns out to be difficult to obtain a replacement as she is also the substitute. She communicates with the authorities by telephone, since they do not come to the school, or come little. The school does not possess a telephone line. She communicates by way on her personal cell phone and it is she who pays with her salary this expense.

She feels that she cannot be absent, and it is the loneliness that most weighs down on her. If something happens to her or the students there is no one who would come to assistance. There are no neighbours nearby. She supports two positions.

If the school is nice, tidy and clean it is because of the personal effort.

With regards to the scale of satisfaction it arises that she feels very satisfied in terms of using her abilities, even if she does not obtain recognition for the excellent work, except by the pupils and the families. Likewise she feels that her suggestions are not listened to. She is consent with work hours, salary, labor stability and possibilities of promotion.

\subsection{The peripheral school}

815 pupils distributed in two shifts compose the population of this school. In this school most students 
come from the suburbs of the city, which is poorer. Those of the neighborhood go to private schools. Some children come without eating. Others, fall asleep in class.

With regards to the building, the school possesses good natural lighting complemented with fluorescent lights; there is noise of the street, the windows are barred, in winter it is cold, and heaters used to warm water begin to work at the end of the winter vacations; in every classroom there are two ceiling ventilators but they do not use them for fear of accidents. Dampness is perceived in the base of the walls, which are as dirty as the furniture. Deterioration is observed in the school. In September the school was flooded while the students and teachers were inside. The windows were jammed and they cannot be opened which means that classrooms become very warm.

With regard to the facilities, there is quite an extent; classrooms, playing fields and the sports ground, a gymnasium, trees in the playgrounds, with a large and small playground existing.

The basic furniture of the classrooms consists of two chalk blackboards, one at the front and other at the back of the class; two cupboards; furniture of wood and premolded plastic; there is a lack of place for books. The office of the teacher is old, of wood with paint peeling, and the teacher's chair is the same as that of the pupils. Many schools have the same aspect of a glorious past and humble present, which gives a sad tone to the school. The maintenance of such an old building becomes very expensive for the financial support that they receive both from the government and from the parental associations. Stuck to the walls appear to be illustrations and works of the pupils.

With regard to the labor relations, teachers say that as director's change, their leadership style changes too and they need to adapt. Some of them are very ancient in the post and they continue to have a job post qualified as substitute. "Most of the medical absences are due to tiredness, caused by problems of behavior of the pupils" say the teachers. When there is a "problem" child, they are not taken into consideration. "The teacher detects problems of behavior, the child is sent to office of the educational psychologist that does the evaluation, and then this diagnose goes on to Direction of Psychology of the Occupational Health Department that should accept the diagnose and then the father also has to accept that the child should begin a treatment. If the parents do not accept that the child should do a treatment, it is not possible to do anything. This process normally lasts two years and in this time there are two the teachers who have this child in the class, and do not manage to solve anything. In general the only solution that we manage to find is to the change the pupil of school."

The Director of the establishment says to be in charge of the management, direction and functioning of different aspects of the school, for example matters of infrastructure.

She explains certain differences between classes: "the younger have only Sports, whereas the older have Sports, English, Music, Dance and Drawing. We see here a problem since the stimulation instead of being early only appears in the second cycle and the first one has nothing except for Sports."

The parent is summoned by the educational psychologist or herself either telephonically or by means of a note. There is a meeting with the secretary to whom all the paperwork/forms is presented. Also with the vicedirector and the inspector.

School acts take place in two shifts and parents like the director to attend both. Students get on very well and disicplinary issues are not abundant among them. Parents have power/are of importance and if they consider the answer they get from the school insatisfactory they contact the media. It is vital to know how to deal with conflict.

The staff under supervision of the principal consists of 48 people. Relationship with the community also plays a central part for the school. Shop owners in the neighborhood are former students/used to attend that school and help the way they can. After the flood they organized a festival on a Sunday and they raised funds for the Garrahan Children's Hospital. They collect/donate clothes for the school as well.

"Everybody looks at this school/keeps an eye on this school, it's a place everybody look at. I have a clear idea of the type of student we expect to develop/form" the director says.

The auxiliary states she suffers from LBP, pain in her legs as she carries/moves desks/tables and chairs.

She says "When it's flooded there's more work to $d o$ ". At the present there are 3 auxiliaries, there used to be 5 of them but now there's two vacant positions. She walks much, goes up and down the stairs, the amount of work to do is everyday bigger. She cleans the director's office, the secretary's office, the teacher's room/hall and the video room. She also gets the door, welcomes people who visit the school, makes photocopies, does the groceries for the school, etc.

\subsection{The centric school}

It's a big school in the city which 600 students at- 
tend. The building is shared with the secondary school which works in the upper floor. The ground floor is exclusive to the primary school.

The school has wide spaces of traffic and walls re-dressed in ceramics. There is a good condition of conservation though spots of dampness are observed in several places. We find bathrooms for pupils in good conditions, that they remain opened during the whole day. They were rebuilt a year ago, since previously they had the ancient system of lavatories.

The classrooms possess radiant heaters, ventilators, natural and artificial lighting with fluorescent pipes without grid, green blackboards. Much noise comes from the playground and the street. The bell rings frequently and in a high pitch, it can also be heard from the classroom. At nights an evening school for adults works in the same building.

It is worth mentioning that classrooms located next to the school buffet get the noise coming from it as well as the one generated in the backyard of the school next-door.

In order to make a better use of the patio, recesses are not simultaneous. The problem, which this system involves, is that while primary students take their recess they make noise and disturb secondary students classes and vice versa.

On occasions, so much noise comes from the other school that classrooms close to it must remain with its windows closed. This produces a hot atmosphere which is specially annoying in summertime.

During our meeting with the teachers they complained about having anxiety issues, they highlighted the social issues that impact in the classroom. They state that there is no training whatsoever for certain positions such as secretary; not all schools count with substitute.

They say that most teachers, especially the youngest, do not have "skin" for the classroom. They suggest that it is necessary to change the educational protocole to avoid the abuse of medical leaves. They report that there are amongst teachers many cases of hormonal pathologies and difficulties to achieve the pregnancy.

The director during his interview says to be the person in charge of everything what happens in the school. She comments that the regulation is very ancient, that is necessary to modify it and to update it.

The director does three meetings with the personnel a year: one in beginning of the year, another one half of year and another one when they finished the year. She joins the personnel of both shifts. The director reports " our work is pedagogic but in cer- tain moments we are firemen the major problem that we have is the absence of the family. "

The major problem is when pupils come in an attitude of defense or attack with their parents. The teacher does not have any specific training in order to deal with this kind of situation.

Every day they remain for one more hour of class to be able to speak with the vicedirector "If you read the regulation its says how to do things in general but there are details that you have to work out by yourself."

The vicedirector says that it is necessary to work outside of school hours as well. She must go to the school board regularly, the fifth working day of the month she has to collect the pay cheques for those who have begun recently and as they don't get paid directly into their bank account yet; they also assist in the Head office health inspection by delivering the pupils' chickenpox certificates; withdrawal certificates, and pupils' school reports.

The secretary is the link between teacher and the school directors. A copy of the student's school ID card, provided by computer, is also held by the secretary, as well as the file of every pupil, which also must be input on to computer. The pupil's school file has 5 pages. Information is also required to be updated. Also undertaken are courses on regulation. The teacher does the record (once a month) since there are 19 courses in total there are 19 records to make each month.

As we can see, the amount of tasks is large and varied. Here we only tell a small part of the tale. The real finding of the work, determined by the statements of the workers, is the way in which the role of the teacher takes a completely new direction at the moment when the directors and teachers undertake their work. The new social phenomena that influence so greatly the life of the pupils and the teachers means that this role must be re-read and newly interpreted year after year to confront new challenges and to thus assure the good development of the classes.

\section{Discussion}

During this preliminary trial we have been able to adjust the instruments to advance with the definitive sample of the schools of the province of Buenos Aires. Nevertheless, as ergonomists, what we consider to be essential to this stage was the ability to successful reformulate the requirements of the job profiles to make an intervention more participative and centered 
on a workers' point of view and not in our view as experts who knows the work of others. On the other hand, we also believe that it is central to this first stage of the research the power to recuperate the voice of the workers and to make clear the wide variability of real situations that lead the workers to deploy unique skills to assure the education in each of these schools. " To be a teacher " is not the same thing in a rural school, a peripheral school and a central school. The challenges are different. The factors of reality are different.

The pupils, the parents, the community, the teachers present their own logic and this leads the workers in the sector of education to have to adapt in a systematic way to the new changes. There is no way of statically regulating the task of the teacher. The ability to collectively fight with the reality appears therefore as the principal challenge. The institutional toing and froing of the government, the cooperative association, and the neighborhood will play a key role in this process.

The management of the different schools is widely varied. On having advanced with the project, this understanding has even deepened: schools in the river-delta, very large schools and schools in situations with pupils in situation of great social precariousness, very young teachers and directors, or almost retiring. Undoubtedly, the prescription of the task of school workers does not reflect the work that these workers actually realize.

Amongst the challenges that arise is that of the limit of the labor activity inside and out of the work hours. Preparing classes, creating binds with the community or working with the parents associations are also part of the school workers' activity. If the workers limited themselves to doing what is written in the regulation, the schools would not be able to educate.

The levels of violence in the population of pupils, parents and even between the workers can reach very high levels and can generate conflicts that impede or even prevent the teachers from realizing their job. In this respect, suffering and psychosocial factors assume in the educational sector a central dimension. In those schools where the relations of cooperation between the actors can be established a great part of the difficulties presented by reality will be solved. Nevertheless, the aptitude to adapt collectively to the new challenges will not always be met and there new conflicts will emerge and even more number of withdrawals, leaves of absence and sickness amongst the work team.
The assessment methods of educational workers are not unified in all the establishments. This being the case, the capacity of the directors to establish collective and individual clear goals will be one of the central elements to obtain good levels of performance both of the teachers and of the pupils.

The questions of gender influence greatly in Argentina's Education System, where the majority of these workers are women. The risks to which they are exposed, physically and due to aggression are very large. The social pressures to struggle with the housekeeping or the care of their own children appear as a source of worry and dilemma at the moment of going to the work.

Many are the new challenges in the new context in which school workers look to assure education. This preliminary trial allowed us to reformulate the request in order to show how teachers, directors and auxiliaries deal with these matters in order to get a wider approach of the work situation.

\section{References}

[1] Dejours, Christophe. Trabajo y sufrimiento. La banalización de la injusticia social. Modus Laborandi. Madrid, 2009.

[2] Return N., Mazeau, M. (1987) L'enfant au travail. Pour une ergonomie scolaire, Revue des conditions de travail $n{ }^{\circ} 29$, mai - juin 1987.

[3] Guerin, F., Laville, A., Daniellou, F., Duraffourg, J., Kerguelen, A., Comprender el trabajo para transformarlo. La práctica de la Ergonomía. Modus Laborandi, Madrid, 2009.

[4] Lancry-Hoestland A., Six F., Furon D., (1990) - Ergonomie scolaire - Paris: Edithions Techniques - Encycl. Phys. Chir., A10 16795.

[5] Leal Ferreira, Leda ; de Araujo, Tânia Maria; Lopes Batista, José Hélio. O Trabalho de professores na Educaçao Básica na Bahia. Fundacentro. Sao Paulo, 2009. 